\title{
Constituintes químicos, fenóis totais e atividade antioxidante de Sterculia striata St. Hil. et Naudin
}

\author{
Danielly Albuquerque COSTA ${ }^{1}$, Mariana Helena CHAVES², Wisllan Cesar Santos SILVA³, Charllyton Luis \\ Sena COSTA ${ }^{4}$ \\ RESUMO \\ A investigação fitoquímica das cascas do caule de Sterculia striata St. Hil. et Naudin, através de métodos cromatográficos, \\ conduziu ao isolamento dos esteróides sitosterol, estigmasterol e sitosterol-3-O- $\beta$-D-glicopiranosídeo, além de quatro \\ triterpenóides pentacíclicos, o lupeol, 3- $\beta$-O-acil lupeol, lupenona e ácido betulínico. As estruturas desses compostos foram \\ identificadas por análise dos espectros de $\mathrm{RMN}{ }^{1} \mathrm{H}$ e ${ }^{13} \mathrm{C}$ e comparaçóes com dados da literatura. Para determinação do teor \\ de fenóis totais do extrato etanólico de S. striata utilizou-se o reativo Folin Ciocalteu, enquanto na avaliação da atividade \\ antioxidante empregou-se o radical livre DPPH. Este é o primeiro trabalho descrevendo o estudo químico com as cascas do \\ caule desta espécie.
}

PALAVRAS-CHAVE: Sterculia striata, esteróides, triterpenóides, atividade antioxidante.

\section{Chemical constituents, total phenolics and antioxidant activity of Sterculia striata St. Hil. et Naudin}

\section{ABSTRACT}

The phytochemical investigation of the stem bark of Sterculia striata St. Hil. et Naudin by chromatographic methods led to the isolation of sitosterol, stigmasterol and sitosterol-3-O- $\beta-\mathrm{D}$-glucopyranoside, besides pentacyclic triterpenoids, lupeol, $3-\beta$-O-acyl-lupeol, lupenone and betulinic acid. The structures of these compounds were identified by ${ }^{1} \mathrm{H}$ and ${ }^{13} \mathrm{C}$ NMR spectral data analysis and comparison with literature data. For determining of the phenolic content of the etanolic extract of Sterculia striata we used the Folin Ciocalteu reagent, and for the evaluation of antioxidant activity, we utilized the DPPH free radical. This is the first work reporting the chemical study with the stem bark of this species.

KEYWORDS: Sterculia striata, steroids, triterpenoids, antioxidant activity.

\footnotetext{
1 Universidade Federal do Piaúi. E-mail: ac_danielly@hotmail.com

2 Universidade Federal do Piauí. E-mail: mariana@ufpi.edu.br

3 Universidade Federal do Piauí. E-mail: wisllancss@hotmail.com

${ }^{4}$ Universidade Federal do Piauí. E-mail: charllysena@hotmail.com
} 


\section{INTRODUÇÃO}

As famílias Sterculiaceae, Tiliaceae e Bombacaceae foram recentemente reclassificadas com base em dados filogenéticos da APG, passando a compor a família Malvaceae (APG, 2003). Esta família é constituída por 243 gêneros e 4225 espécies distribuídas por quase todo o mundo, com exceção de regióes muito frias, ocorrendo predominantemente nas regiōes tropicais, principalmente na América do Sul (Stevens, 2003; Heywood, 1993). Neste trabalho realizou-se o estudo fitoquímico de Sterculia striata no intuito de reforçar a sua inclusão nesta família, através da comparação de constituintes químicos isolados com aqueles relatados na literatura para outras espécies de Malvaceae. Adicionalmente foi realizada a avaliação do conteúdo de compostos fenólicos e a atividade antioxidante para o extrato etanólico desta espécie.

Sterculia striata, conhecida popularmente no Brasil como chichá, amendoim-da-mata e castanha-de-macaco (Almeida et al., 1998), ocorre do Amazonas ao Piauí, Mato Grosso, Minas Gerais e Rio Grande do Sul (Pio Corrêa, 1974). A espécie é uma árvore ornamental, que fornece amêndoas, consumidas pela população, nas formas cruas, cozidas ou torradas e pela fauna, ao natural (Lorenzi et al., 1996). Na medicina popular, suas folhas têm sido empregadas topicamente com manteiga quente ou óleo de oliva para o tratamento de furúnculos (Agra et al., 2007). Além disso, é considerada uma das árvores importantes na fabricaçáo de papel e fornece madeira branca, macia e leve, própria para o forro de mobílias, obras internas, palito de fósforo, molduras e caixas (Pio Corrêa, 1974). Na literatura existem apenas dois trabalhos fitoquímicos com as sementes desta espécie, destacando a presença de ácidos graxos ciclopropenoídicos, impróprios para consumo humano (Aued-Pimentel et al., 2004; Chaves et al., 2004). Apesar disso, o óleo da semente pode ser aproveitado na preparaçáo de biodiesel (Araújo, 2008).

Investigaçôes fitoquímicas de outras espécies de Sterculia mostraram um perfil químico diversificado, com isolamento de flavonóides, alcalóides, outros compostos fenólicos e ácidos graxos (Ranganathan e Nagarajan, 1980; Anjaneyulu e Raju, 1987; Hayman et al., 1988).

Atualmente existe um grande interesse no estudo dos antioxidantes por serem substâncias que em pequenas quantidades podem prevenir e apresentar alto potencial terapêutico de doenças causadas por radicais livres (Noguchi e Niki, 2000). Os radicais livres de oxigênio ou mais genericamente espécies reativas de oxigênio são produtos do metabolismo normal das células, os quais estão associados a processos como produção de energia, fagocitose, regulação do crescimento celular, sinalização intercelular e síntese de substâncias biológicas importantes. No entanto, seu excesso apresenta efeitos prejudiciais, tais como a peroxidação dos lipídeos de membrana e agressão às proteínas dos tecidos e das membranas, às enzimas, carboidratos e DNA (Hussain et al., 1987). Dessa forma, os radicais livres encontramse relacionados com várias patologias, tais como: artrite, choque hemorrágico, doenças cardíacas, catarata, disfunçôes cognitivas, envelhecimento e câncer, podendo ser a causa ou o fator agravante do quadro geral (Halliwell et al., 1995). Estas observações têm estimulado a busca de novas substâncias fitoquímicas com potencial antioxidante.

O interesse pela determinação do conteúdo de compostos fenólicos e avaliaçáo da atividade antioxidante da $S$. striata foi reforçado pela presença de substâncias flavonoídicas e alcaloídicas relatadas em outras espécies do gênero Sterculia $e$ pela inexistência na literatura de estudo semelhante sobre a espécie.

\section{MATERIAL E MÉTODOS}

\section{PROCEDIMENTOS EXPERIMENTAIS GERAIS}

Todos os solventes usados eram analiticamente puros (P.A.) obtidos das marcas Vetec, Quimex ou Synth. Para as cromatografias em coluna utilizou-se como fase estacionária gel de sílica 60 (Merck) 7734 (partículas com 0,063-0,2 mm), tendo como suporte colunas de vidro com dimensóes variando de acordo com a quantidade de amostra a ser purificada. A cromatografia em camada delgada comparativa (CCDC) foi empregada para análise e reunião das fraçôes obtidas por cromatografia em coluna, sendo preparadas por uma suspensấo de gel de sílica $60 \mathrm{G}$ Vetec com água destilada (1:1). As revelaçôes destas cromatoplacas foram feitas por borrifamento com soluçáo de sulfato cérico e o critério de pureza adotado para as substâncias isoladas foi a observaçáo de uma única mancha em CCDC, em diferentes sistemas de eluentes.

O reagente Folin-Ciocalteu foi adquirido da Merck, o radical DPPH (2,2-difenil-1-picril-hidrazila) e o BHT (butil-hidroxi-tolueno) da Sigma-Aldrich e o ácido gálico da Vetec.

As medidas de absorção foram feitas usando espectrofotômetro UV-Vis Hytachi U-3000. Os espectros de RMN de ${ }^{1} \mathrm{H}$ e de ${ }^{13} \mathrm{C}$ foram obtidos em espectrômetros Brüker modelos Avance DRX-500 (UFC) e DRX 400 (UFAL), operando respectivamente a 500 e $400 \mathrm{MHz}\left({ }^{1} \mathrm{H}\right)$, 125 e $100 \mathrm{MHz}\left({ }^{13} \mathrm{C}\right)$, utilizando $\mathrm{CDCl}_{3}$ como solvente e TMS como referência interna.

\section{MATERIAL VEGETAL}

As cascas do caule de Sterculia striata foram coletadas na fazenda Várzea da Cruz, município de Oeiras-PI, em novembro de 2004. A identificação foi realizada no Herbário Graziela Barroso - UFPI, onde se encontra depositada uma exsicata da espécie, codificada como TEPB 10165. 


\section{EXTRAÇÃO, FRACIONAMENTO E ISOLAMENTO DOS CONSTITUINTES QUÍMICOS}

O material vegetal $(1,8 \mathrm{~kg})$ foi seco, moído e extraído por maceração com etanol. A soluçẫo etanólica foi concentrada em evaporador rotatório a $60^{\circ} \mathrm{C}$, produzindo $67,0 \mathrm{~g}$ do extrato etanólico bruto. Uma parte deste material $(25,0$ g) foi submetido a uma coluna filtrante, usando como solventes hexano, diclorometano, acetato de etila e metanol sucessivamente, resultando em suas respectivas fraçóes, após evaporação dos solventes.

A fraçáo diclorometânica $(5,0 \mathrm{~g})$ foi submetida à cromatografia em coluna de gel de sílica 60 e eluída com hexano, acetato de etila e metanol, em gradiente crescente de polaridade, resultando em 125 fraçóes de $125 \mathrm{~mL}$ cada, as quais foram concentradas em evaporador rotatório e reunidas de acordo com seus fatores de retenção em nove grupos. O grupo S2 (2,7 g) formado pelas fraçóes 02-07, eluídas com hexano-AcOEt (95:5), foi posteriormente submetido a nova cromatografia em coluna sob gel de sílica com hexano$\mathrm{CH}_{2} \mathrm{Cl}_{2}$, em gradiente crescente de polaridade, fornecendo as substâncias $\mathbf{1}(10 \mathrm{mg}), \mathbf{2}(80 \mathrm{mg})$ e $\mathbf{3}(1,3 \mathrm{~g})$.

Os grupos S5 (770 mg, fraçóes 27-44), eluído com hexano-AcOEt (9:1), e S8 (305 mg, fraçôes 67-78), eluído com hexano-AcOEt (8:2), após recristalização em metanol, forneceram a mistura dos esteróides $\mathbf{4 a}$ e $\mathbf{4 b}(200 \mathrm{mg})$ e a substância $5(22 \mathrm{mg})$, respectivamente.

Durante o fracionamento do extrato etanólico em coluna filtrante, na fração eluída com acetato de etila formou-se um precipitado, que após suspensão em metanol seguido de filtração resultou na substância $6(60 \mathrm{mg})$.

\section{PREPARAÇÃO DO DERIVADO ACETILADO DA SUBSTÂNCIA 6}

Uma alíquota da substância $6(25 \mathrm{mg})$ foi acetilada através da adição de $2 \mathrm{~mL}$ de anidrido acético e $1 \mathrm{~mL}$ de piridina, em presença de quantidade catalítica de DMAP (4-dimetilaminopiridina), permanecendo em repouso por $12 \mathrm{~h}$. Após esse período, a mistura reacional foi vertida sobre água destilada gelada em funil de decantaçáo e extraída com $\mathrm{CHCl}_{3}(4 \times 10 \mathrm{~mL})$. A solução clorofórmica foi lavada com soluçâo de $\mathrm{CuSO}_{4} 5 \%,(3 \times 20 \mathrm{~mL})$, posteriormente com água destilada $(2 \times 20 \mathrm{~mL})$ e seca com $\mathrm{Na}_{2} \mathrm{SO} 4$ anidro. Após a remoçáo do solvente em evaporador rotatório obteve-se o produto acetilado, codificado como $\mathbf{6 a}(40 \mathrm{mg})$.

\section{DETERMINAÇÃO DE FENÓIS TOTAIS}

O teor de fenóis totais presentes nas amostras do extrato etanólico de $S$. striata foi medido em triplicata utilizando-se um espectrofotômetro na região do visível, o reagente FolinCiocalteu e padrão de ácido gálico conforme a metodologia anteriormente descrita (Sousa et al.; 2007). Os resultados foram expressos em mg de EAG (equivalentes de ácido gálico) por g de amostra. A equação da curva de calibração do ácido gálico foi $C=809,0200 A+5,0827$, onde $C$ é a concentração do ácido gálico, $A$ é a absorbância a $750 \mathrm{~nm}$ e o coeficiente de correlação $R=0,999$.

\section{ATIVIDADE ANTIOXIDANTE}

A avaliação da atividade antioxidante do extrato EtOH da S. striata foi determinada pela sua capacidade de seqüestrar o radical livre DPPH segundo metodologia descrita por Sousa et al. (2007). As medidas do decréscimo da absorbância das soluçōes testadas foram feitas em espectrofotômetro UVVis no comprimento de onda $516 \mathrm{~nm}$, utilizando-se como controle positivo o BHT. Todas as análises foram realizadas em triplicata.

Soluçôes do extrato EtOH e do BHT foram preparadas em metanol nas concentraçóes de 250, 200, 150, 100, 50 e 25 $\mu \mathrm{g} / \mathrm{mL}$. Foi utilizado como branco uma mistura de $0,3 \mathrm{~mL}$ da solução metanólica do extrato ou do controle positivo com 2,7 $\mathrm{mL}$ de metanol. As misturas reacionais consistiram de $0,3 \mathrm{~mL}$ de solução metanólica do extrato ou controle positivo com 2,7 $\mathrm{mL}$ da solução estoque de DPPH na concentração de $40 \mu \mathrm{g} /$ $\mathrm{mL}$. Os valores de absorbâncias destas misturas foram medidos no $1^{\circ}, 5^{\circ}$ e $10^{\circ} \mathrm{min}$, e a cada $10 \mathrm{~min}$ até completar $1 \mathrm{~h}$.

A porcentagem de atividade antioxidante (\% AA) foi determinada a partir dos valores de absorbância de todas as concentraçóes testadas no tempo de $30 \mathrm{~min}$, conforme a equação:

$\% \mathrm{AA}=\left\{\left[\mathrm{Abs}_{D P P H}-\left(\mathrm{Abs}_{\text {amostra }}-\mathrm{Abs}_{\text {branco }}\right)\right]\right\} \times 100 / \mathrm{Abs}_{D P P H}$

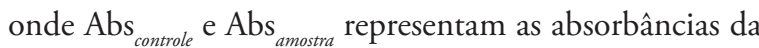
solução metanólica de DPPH e da mistura reacional (amostra + DPPH), respectivamente.

\section{RESULTADOS E DISCUSSÃO}

O estudo fitoquímico das cascas do caule de $S$. striata conduziu ao isolamento e identificação de quatro triterpenos $(1,2,3$ e 5$)$ e três esteróides, sendo dois destes, isolados
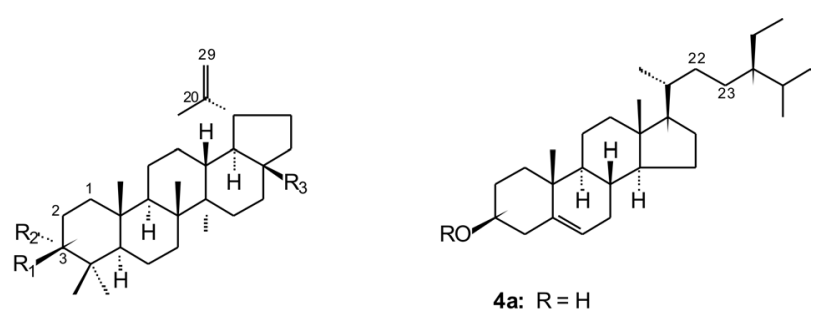

$$
\begin{aligned}
& \text { 1: } \mathrm{R}_{1}=\mathrm{CH}_{3}\left(\mathrm{CH}_{2}\right) \mathrm{nCOO} ; \mathrm{R}_{2}=\mathrm{H} ; \mathrm{R}_{3}=\mathrm{CH}_{3} \\
& \text { 2: } \mathrm{R}_{1}=\mathrm{R}_{2}=\mathrm{O} ; \mathrm{R}_{3}=\mathrm{CH}_{3} \\
& \text { 3: } \mathrm{R}_{1}=\mathrm{OH} ; \mathrm{R}_{2}=\mathrm{H} ; \mathrm{R}_{3}=\mathrm{CH}_{3} \\
& \text { 5: } \mathrm{R}_{1}=\mathrm{OH} ; \mathrm{R}_{2}=\mathrm{H} ; \mathrm{R}_{3}=\mathrm{COOH}
\end{aligned}
$$

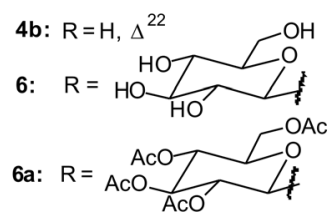

Figura 1 - Substâncias isoladas das cascas do caule de S. striata 
como mistura $(4 a+4 b)$ e o outro na forma glicosilada (6) (Figura 1).

O espectro de RMN de ${ }^{1} \mathrm{H}$ da substância 1 mostrou sinais entre $\delta_{\mathrm{H}} 0,73-0,98$ e a 1,62 característicos de grupos metila em carbono não hidrogenado de triterpenóides, sendo este último, ligado a carbono $\mathrm{sp}^{2}$, além de um dupleto em $\delta_{\mathrm{H}} 4,63(\mathrm{~J}=2,0$ $\mathrm{Hz})$ e um multipleto em $\delta_{\mathrm{H}} 4,51$, atribuídos a hidrogênios de grupo vinilidênico (H-29a e H-29b) em triterpenóide de esqueleto lupano. A desblindagem do H-3 em relação ao observado em triterpenóides $3-\beta-\mathrm{OH}$, evidenciada pelo duplo dupleto em $\delta_{\mathrm{H}} 4,42(\mathrm{~J}=11$ e $5 \mathrm{~Hz})$, sugeriu que a hidroxila da posição 3 foi esterificada por um ácido. $\mathrm{O}$ sinal intenso em $\delta_{\mathrm{H}} 1,20$ e os tripletos em 0,80 e em 2,22 caracterizaram a presença de um éster graxo (Abreu et al., 2001). O espectro de $\mathrm{RMN}$ de ${ }^{13} \mathrm{C}$ corroborou com os dados anteriores ao apresentar sinais em $\delta_{C}$ 150,9 (C-20) e 109,4 (C-29), típicos do esqueleto lup-20(29)-eno (Olea e Roque, 1990). Adicionalmente, apresentou sinal de carbono carboxílico de éster em $\delta_{\mathrm{C}} 173,4$, além de outros sinais, que evidenciaram os efeitos de blindagem para o sinal do C-2 $(\Delta \delta=3,5 \mathrm{ppm})$ e desblindagem para o $\mathrm{C}-3$ ( $\Delta \delta=1,7 \mathrm{ppm})$, em comparação aos respectivos carbonos em triterpenóides $3-\beta-\mathrm{OH}$. Estes dados mostraram-se consistentes com a presença de um éster de lupeol (Abreu et al., 2001; David et al., 2003).

$\mathrm{O}$ espectro de RMN de ${ }^{1} \mathrm{H}$ da substância 2 mostrou sinais característicos de triterpenóides entre $\delta_{\mathrm{H}}$ 0,67-1,92, além de dois simpletos largos em $\delta_{\mathrm{H}} 4,57$ e 4,70, típicos de ligação dupla gem-dissubstituída em esqueleto lupano. A presença no espectro de $\mathrm{RMN}$ de ${ }^{13} \mathrm{C}$ de um sinal em $\delta_{\mathrm{C}}$ 218,4 característico de carbonila, e de sinais em $\delta_{C} 109,1$ e 150,7 para carbonos olefínicos, associados à comparaçóes com dados descritos na literatura permitiu identificá-la como sendo a lupenona (Mutai et al., 2004; Olea e Roque, 1990).

As substâncias 3 e $\mathbf{5}$ apresentaram em seus espectros de $\mathrm{RMN}$ de ${ }^{1} \mathrm{H}$ sinais semelhantes aos observados para a substância 2, e mostraram ainda, um sinal adicional em $\delta_{\mathrm{H}} 3,20$, característico de hidrogênio oximetínico em triterpenóides $3 \beta-\mathrm{OH}$. Os espectros de $\mathrm{RMN}$ de ${ }^{13} \mathrm{C}$ das substâncias 3 e 5 fortaleceram a proposta anterior ao exibir um sinal em $\delta 79,0$, confirmando a estereoquímica $3 \beta-\mathrm{OH}$. O esqueleto do tipo lupano para estas substâncias foi definido pelos sinais característicos em $\delta_{\mathrm{C}} 109,3\left(\mathrm{CH}_{2}\right)$ e $150,9(\mathrm{C})$. O espectro de RMN de ${ }^{13} \mathrm{C}$ da substância 5 apresentou um sinal a menos de carbono metílico que o observado para as substâncias 2 e 3, bem como, um sinal em $\delta_{\mathrm{C}} 180,5$ atribuído a carbonila de ácido. Comparaçôes dos deslocamentos de RMN de ${ }^{13} \mathrm{C}$ de 3 e 5 com a literatura (Olea e Roque, 1990; Mahato e Kundu, 1994; Castilho e Kaplan, 2008) permitiram identificá-las como o lupeol e o ácido betulínico, respectivamente, sendo o lupeol o constituinte majoritário da espécie estudada.

$\mathrm{O}$ espectro de RMN de ${ }^{1} \mathrm{H}$ da mistura das substâncias $\mathbf{4 a}$ e $\mathbf{4 b}$ apresentou sinais entre $\delta_{\mathrm{H}} 0,62-2,40$ correspondentes a grupos metílicos de esteróides, um multipleto em $\delta_{\mathrm{H}} 3,54$ (H-3) e sinais em $\delta_{\mathrm{H}} 5,02,5,16$ (dd, H-22 e H-23, 4b) e $5,36(d l, H-6, \mathbf{4 a + 4 b})$ de hidrogênios olefínicos. Estes dados, aliados à análise do espectro de $\mathrm{RMN}$ de ${ }^{13} \mathrm{C}$ da mistura e comparação com dados da literatura (Goulart et al., 1993; Ahmed et al., 1992) permitiram identificar as estruturas do sitosterol (4a) e estigmasterol (4b).

A substância 6 foi identificada a partir do seu derivado acetilado (6a), obtido após tratamento com anidrido acético e piridina. O espectro de $\mathrm{RMN}$ de ${ }^{1} \mathrm{H}$ de $6 a$ apresentou sinais semelhantes aos observados para a mistura $4 \mathrm{a}$ e $4 \mathrm{~b}$, e sinais adicionais entre $\delta_{\mathrm{H}}$ 3,50-5,18 típicos de açúcar e entre $\delta_{\mathrm{H}}$ 1,90-2,10 de hidrogênios metílicos de grupos acetato (Alves et al., 2001). A análise do espectro de RMN de ${ }^{13} \mathrm{C}$ permitiu identificar, sinais característicos do sitosterol, um de carbono anomérico $\left(\delta_{\mathrm{C}} 100,0\right)$, cinco de carbono carbinólico entre $\delta_{\mathrm{C}} 62,82$ e 71,8 e um em $\delta_{\mathrm{C}} 80,0$ compatível com o deslocamento químico do C-3 de esteróide glicosilado (Alves et al., 2001). Os grupos acetoxílicos foram confirmados neste espectro pelos sinais entre $\delta_{\mathrm{C}} 20,6-20,7\left(\mathrm{CH}_{3}\right)$ e $\delta_{\mathrm{C}} 170,3$ $170,4(\mathrm{C}=\mathrm{O})$. Os dados do produto acetilado em comparação com os relatados na literatura (Alves et al., 2001; Macari et al., 1990) permitiram confirmar a presença 3-O- $\beta-D-$ glicopiranosilsitosterol (6).

\section{DETERMINAÇÃO DE FENÓIS TOTAIS}

O teor de fenóis totais, determinado pelo método FolinCiocalteau, para o extrato etanólico de S. striata foi de 63,94 $\pm 5,59 \mathrm{mg}$ de equivalentes de ácido gálico (EAG) por grama de amostra, um valor considerado baixo quando comparado aos extratos etanólicos de outras espécies descritas na literatura (Sousa et al., 2007; Kähkönen et al., 1999). Portanto, pode-se concluir que a espécie estudada possui poucos flavonóides, alcalóides, taninos ou outras substâncias fenólicas.

\section{ATIVIDADE ANTIOXIDANTE}

O resultado da avaliaçáo da atividade antioxidante (\% AA) do extrato etanólico de $S$. striata (cascas do caule) e do controle positivo BHT, determinada pelo ensaio de DPPH, nas diferentes concentraçóes $(25-250 \mu \mathrm{g} / \mathrm{mL})$ está apresentado na Figura 2.

O extrato EtOH de S. Striata, em todas as concentraçóes testadas, exibiu valores de atividade antioxidante inferiores a $50 \%$, inviabilizando a determinação da $\mathrm{CE}_{50}$ (concentração eficiente capaz de reduzir o DPPH em 50\%). Ao ser comparado com o padrão BHT no tempo de 30 min de reaçáo, apresentou uma fraca atividade antioxidante, pois na maior concentração 


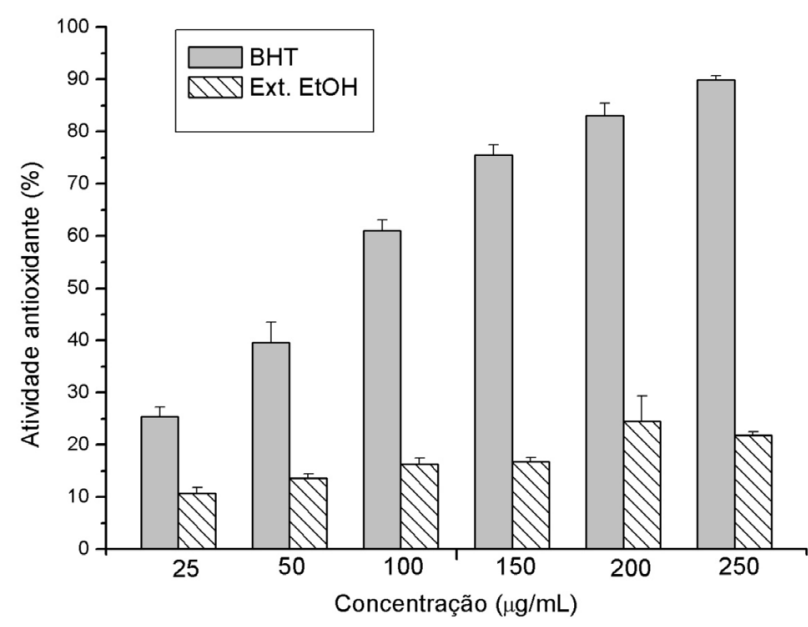

Figura 2 - Porcentagem de atividade antioxidante do extrato etanólico de $S$. striata e do BHT no tempo de 30 min.

testada $(250 \mu \mathrm{g} / \mathrm{mL})$ o percentual de atividade foi de $21,19 \pm$ $0,78 \%$, comparável ao obtido para o $\operatorname{BHT}(25,41 \pm 1,82 \%)$ na menor concentração $(25 \mu \mathrm{g} / \mathrm{mL})$.

\section{CONCLUSÃO}

O estudo fitoquímico de $S$. striata conduziu ao isolamento de três esteróides e quatro triterpenos pentacíclicos de esqueleto lupano. $\mathrm{O}$ isolamento destas substâncias contribuiu para o estudo quimiotaxonômico da família Malvaceae e está parcialmente de acordo com o perfil químico esperado de plantas do gênero Sterculia, considerando que não foi constatada a ocorrência de flavonóides, alcalóides ou outros compostos fenólicos, substâncias que têm, reconhecidamente, propriedades antioxidantes. Este fato justifica a fraca atividade antioxidante apresentada para o extrato etanólico da espécie, que foi compatível com o baixo teor de fenóis totais.

O sitosterol e o estigmasterol já haviam sido identificados anteriormente nesta espécie, em estudo realizado com as sementes, entretanto o éster de lupeol e o ácido betulínico estão sendo relatados pela primeira vez no gênero Sterculia. Este é o primeiro trabalho descrevendo o estudo químico com as cascas do caule da $S$. striata.

\section{AGRADECIMENTOS}

Ao CNPq, FAPEPI e UFPI pelas bolsas de pesquisa concedidas e apoio financeiro. Ao Centro Nordestino de Aplicação e Uso de Ressonância Magnética Nuclear (CENAUREMN/UFC) e UFAL pela obtenção dos espectros de RMN de ${ }^{1} \mathrm{H}$ e ${ }^{13} \mathrm{C}$ das substâncias.

\section{BIBLIOGRAFIA CITADA}

Abreu, A.S.; Barbosa, P. S.; Müller, A. H.; Guilhon, G. M. S. P. 2001. Constituintes químicos do caule e das cascas de Croton pullei var. Glabrior (Euphorbiaceae). Revista Virtual de Iniciação Acadêmica da UFPA, 1: 1-9.

Agra, M. F.; Freitas, P. F.; Barbosa-Filho, J. M. 2007. Synopsis of the plants known as medicinal and poisonous in Northeast of Brazil. Revista Brasileira de Farmacognosia, 17: 114-140.

Ahmed, W.; Ahmad, Z.; Malik, A. 1992. Stigmasterol galactoside from Rhynchosia minima. Phytochemistry, 31: 4038-4039.

Almeida, S. P.; Proença, C. E. B.; Sano, S. M.; Ribeiro, J. F. 1998. Cerrado: espécies vegetais úteis. Planaltina: Embrapa, 464 pp.

Alves, C. C. F.; Cranchi, D. C.; Carvalho, M. G.; Silva, S. J. 2001. Triterpenos, esteróide glicosilado e alcalóide isolados de Simira glaziovii. Floresta e Ambiente, 8: 174-179.

Anjaneyulu, A. S. R.; Raju, S. N. 1987. Terpenoids and phenolics from the bark and heartwood of Sterculia urens Roxb. Journal of the Indian Chemical Society, 64: 323-324.

Araújo, F. D. S. 2008. Potencialidade das espécies Jatropha curcas L., Bombacopsis glabra (Pasq.) A. Robyns e Dipteryx lacunifera Ducke para produção de biodiesel. Centro de Ciências da Natureza/ Universidade Federal do Piauí, Teresina, Piauí. 127pp.

Aued-Pimentel, S.; Lago, J. H. G.; Chaves, M. H.; Kumagai, E. E. 2004. Evaluation of a methylation procedure to determine cyclopropenoids fatty acids from Sterculia striata St. Hil. et Nauds seed oil. Journal of Chromatography A, 1054: 235-239.

Castilho, R. O.; Kaplan, M. A. C. 2008. Constituintes químicos de Licania tomentosa Benth.Chrysobalanaceae. Quimica Nova, 31: 66-69.

Chaves, M. H.; Barbosa, A. S.; Moita Neto, J. M.; Aued-Pimentel, S.; Lago, J. H. G. 2004. Caracterização química do óleo de amêndoa de Sterculia striata St. Hil. Et. Naud. Química Nova, 27: 404-408.

David, J. M.; Santos, F. A.; Guedes, M. L. S.; David, J. P. 2003. Flavonóide e triterpenos de Stigmaphyllom paralias. Química Nova, 26: 484-487.

Goulart, M. O. F.; Sant'Ana, A. E. G.; Lima, R. A.; Cavalcante, S. H.; Carvalho, M. G.; Braz-Filho, R. 1993. Fitoconstituintes químicos isolados de Jatropha elliptica. Atribuição dos deslocamentos químicos dos átomos de carbono e hidrogênio dos diterpenos jatrofolonas A e B. Química Nova, 16: 95-100.

Halliwell, B.; Aeschbach, R.; Loliger, J.; Arouma, O. I. 1995. The Characterization of Antioxidants. Food and Chemical Toxicology, 33: 601 .

Hayman, A. R.; Gray, D. O.; Elliot, S. D. 1988. Isolation of histamine from the fruits of Sterculia scaphigera. Fitoterapia, 59: 338

Heywood, V. H. 1993. Flowering plants of world. B. T. London: Batsford Ltda.

Hussain, S. R.; Cillar, J.; Cillard, P. 1987. Hydroxyl radical scavenging activity of flavonoids. Phytochemistry, 26: 2489-2491. 
Kähkönen, M. P.; Hopia, A. I.; Vuorela, H. J.; Rauha, J.; Pihlaja, K.; Kujala, T. S.; Heinonen, M. 1999. Antioxidant Activity of Plant Extracts Containing Phenolic Compounds. Journal of Agricultural and Food Chemistry, 47: 3954-3962.

Lorenzi, H.; Souza, H. M.; Medeiros-Costa, J. T.; Cerqueira, L. S. C.; Von Behr, N. 1996. Palmeiras do Brasil: exóticas e nativas, Plantarum: Nova Odessa, 303 pp.

Macari, P. A. T.; Emerenciano, V. P.; Ferreira, Z. M. G. S. 1990. Identificação dos triterpenos de Miconia albicanstriana através de análise por microcomputador. Quimica Nova, 13: 260-262.

Mahato, S. B.; Kundu, A. P. $1994 .{ }^{13} \mathrm{C}$ NMR spectra of pentacyclic triterpenoids - a complilation and some salient features. Phytochemistry, 37: 1517-1575.

Mutai, C.; Abatis, D.; Vagias, C.; Moreau, D.; Roussakis, C.; Roussis, V. 2004. Cytotoxic lupane-type triterpenoids from Acacia mellifera. Phytochemistry, 65: 1159-1164.

Noguchi, N.; Niki, E. 2000. Forum: Therapeutic Applications of Reactive Oxygen and Nitrogen Species in Human Disease. Free Radical Biology and Medicine, 28: 1538-1546.
Olea, R. S. G.; Roque, N. F. 1990. Análise de misturas de triterpenos por RMN ${ }^{13}$ C. Quimica Nova, 13: 278-281.

Pio Côrrea, M. 1974. Dicionário de plantas úteis do Brasile das exóticas cultivadas. Rio de Janeiro: Ministério da Agricultura.

Ranganathan, R. M.; Nagarajan, S. 1980. Flavonoids of the leaves of Sterculia pallens. Current Science, 49: 309-310.

Sousa, C. M. M.; Silva, H. R.; Vieira-Jr, G. M.; Ayres, M. C. C.; Costa, C. L. S.; Araújo, D. S.; Cavalcante, L. C. D.; Barros, E. D. S.; Araújo, P. B. M.; Brandão, M. S.; Chaves, M. H. 2007. Fenóis totais e atividade antioxidante de cinco plantas medicinais. Química Nova, 30: 351-355.

Stevens, P. F. 2003. Angiosperm phylogeny website. http://mobot. org. Acessado em janeiro de 2006.

The Angiosperm Phylogeny Group 2003. An update of the Angiosperm Phylogeny Group classification for the orders and families of flowering plants: APG II. Botanical Journal of the Linnean Society, 141: 399-436.

Recebido em 27/01/2009

Aceite em 12/05/2009 